\title{
Le Siècle d'Or et la portée idéologique du théâtre espagnol
}

\author{
Strosetzki, Christoph
}

First published in:

Anuario calderoniano 6 (2013), S. 243-257

ISSN: $1888-8046$

() 2013 Vervuert Verlag, Frankfurt am Main 


\title{
LE SIÈCLE D'OR ET LA PORTÉE IDÉOLOGIQUE DU THÉÂTRE ESPAGNOL
}

\author{
Christoph Strosetzki \\ Spanisch-Portugiesisch-Lateinamerikanische Abteilung \\ Romanisches Seminar \\ Universität Münster \\ Bispinghof 3a \\ 48143 Münster \\ stroset@uni-muenster.de
}

[Amuario calderoninno (ISSN: 1888-8046), 6, 2013, pp. 243-257]

Le $\mathrm{XVI}^{e}$ siècle espagnol se caractérise par une mobilité territoriale due à la découverte de l'Amérique, par une mobilité verticale due à l'ascension sociale, et par un exode rural dû à la naissance de nouveaux métiers. Le XvII ${ }^{\mathrm{e}}$ siècle, au contraire, connait selon José Antonio Maravall, une tentative de rétablissement par les monarchies absolues, de la situation ayant existé au Moyen-Âge, à travers tout un appareil de répression et de domination, ainsi que l'utilisation de la culture baroque. Tandis que le théâtre français serait un art pour aristocrates, le thêâtre espagnol serait un art pour le peuple se concentrant sur les émotions des masses. "El teatro español trata de imponer o de man- 
tener la presión de un sistema de poder, y, por consiguente, una estratificación y jerarquía de grupos»'. Maravall soutient que le théâtre sert à des fins propagandistes, qu'il n'argumente pas politiquement mais émotionellement. Le théâtre espagnol, contrairement au théâtre français, n'aurait aucune valeur pédagogique, ne contiendrait aucune sentence édifiante, n'inciterait en aucune façon le spectateur, à travers des exemples, à mener une vie moralement bonne. Les problèmes éthiques ne sont pas posés. "El teatro español es, ante todo, un instrumento político y social, no responde a una preocupación o finalidad ética e incluso es mínima la parte que en él se ocupa de temas religiosos» ${ }^{2}$. Visiblement Maravall ne pense pas à Calderón lorsqu'il refuse de reconnaître le caractère religieux du théâtre du Siècle d'Or. Nous démontrerons à l'aide d'exemples que chez Calderón la situation se présente tout autrement.

Dans ce qui suit nous allons donc réfuter les trois thèses de Maravall par des exemples tirés du théâtre de Calderón. Premièrement nous montrerons que les réflexions, les modèles et les argumentations politiques ne sont absolument pas présentés de manière émotionnelle, mais rationnelle. Ensuite, nous mettrons en évidence quelques questions éthiques et leçons pédagogiques présentes dans le théâtre de Calderón. Enfin nous démontrerons que les sujets religieux y sont traités dans toute leur complexité théologique et que le catholicisme n'y apparaît pas seulement comme un simple modèle d'identification émotionnel.

Mais avant d'étudier quelques argumentations et modèles politiques présentées par Calderón, nous devons interroger brièvement les intentions de Maravall. Ce dernier souligne l'importance au théâtre du thème de l'ascension et de la chute sociales bien qu'il soit conseillé à chacun de rester à la place qui est la sienne dans la société : "Los personajes virtuosos, los que aparecen como ejemplos de la más meritoria moral social, son aquellos que se conforman con su estado, procurando sólo las perfecciones posibles en cada uno de ellos» ${ }^{3}$. Cela correspond à la description aristotélicienne d'un État qui fonctionne et qui serait valable pour l'aristocratie comme pour la démocratie. Les

1 Maravall, 1972, p. 29.

2 Maravall, 1972, p. 31-32.

${ }^{3}$ Maravall, 1972, p. 60. 
drames de Calderón ne sont naturellement pas des drames révolutionnaires dans lesquels serait thématisée la transition d'une forme étatique à une autre, mais ils prennent pour point de départ la société telle qu'elle existe et dans laquelle chacun doit remplir sa fonction. La pensée de Calderón est marquée par Aristote voire par l'aristotélisme néo-scolastique de l'époque qui connaît la démocratie et l'aristocratie, bien que celles-ci soient considérées comme étant de moindre qualité. Dans ce contexte, il est important de noter que toutes ces formes étatiques ont une variante négative: l'ochlocratie, l'oligarchie et la tyrannie. La vigilance est donc de mise face à une monarchie établie qui peut dégénérer en tyrannie. Calderón multiplie les exemples frappants pour montrer comment on peut reconnaître une telle dégénérescence, ce qui y conduit, comment elle peut être évitée, comment réagir face à elle, et pour voir si un sujet a le droit absolu de réagir. Les auteurs de tyrannicides ont prise sur les plus importants événements de l'État. Chez Calderón, leur acte passe pour légitime, bien qu'il émane des différentes basses couches de la société. Dans La gran Cenobia, c'est bien le peuple qui fait la louange du meurtre du tyran.

Tandis que dans la France du XVII siècle, Descartes cherchait la base indubitable d'une connaissance à partir de laquelle il pourrait fonder tout savoir, il existait en Italie et en Espagne à l'époque de la Renaissance et à partir du De rerum inventoribus de Polydore Vergil, des listes qui notifiaient les origines et les innovateurs qui s'étaient penchés sur les différentes disciplines du savoir. La recherche sur les prémisses voire sur les bases du fondement du savoir et de l'action sont donc une question importante pour le début des temps modernes.

Ceci nous conduit à poser aussi pour le théâtre de Calderón les questions fondamentales que nous exposerons dans ce qui suit. Y a-til un état originel qui précède l'état social? Quels sont les rapports entre l'état sauvage présocial et l'état social civilisé? La souveraineté du roi est légitimée par la grâce divine. Si les lois et les ordonnances du roi doivent être conformes à l'esprit d'un créateur divin et guide de l'univers, alors le droit positif doit dériver du droit divin, et la lex positiva dérive de la lex divina. Si le fondement du droit positif est divin, que se passe-t-il lorsque ce droit se détache de son ancrage? Une question similaire est soulevée par Machiavel. Quand le prince agit selon la morale tout en ayant le souci de son maintien au pouvoir, a- 
t-il le droit d'avoir une conduite immorale dans le seul but de mieux conserver son autorité? La raison d'État justifie-t-elle l'absence de morale? Et en quelles circonstances le peuple a-t-il le droit de substituer l'insurrection et le tyrannicide à la morale et à l'obéissance envers le roi? Dans ses pièces Calderón produit une pédagogie par l'exemple, mettant en jeu des actions soit vouées à l'échec, soit couronnées de succès, qu'il recommande d'imiter ou d'éviter.

L'éthique en général a une double importance: d'une part elle est la base de la théorie politique, d'autre part elle fournit les règles du comportement moral individuel. Nous pouvons nous demander si l'acquisition de ce comportement est pratique ou théorique, et si l'ignorance, la violence ou la peur sont des obstacles au bonheur et à la morale. Calderón répond par l'exemple à ces questions, prenant comme arrière-plan le modèle de pensée aristotélicienne de Saint Thomas d'Aquin, lequel a pour point de départ des questions théologiques. À partir de la rétlexion théologique de Calderón, nous chercherons à savoir si la notion chrétienne de Trinité est monothéiste ou polythéiste. Comme nous allons le constater par la suite, le théâtre de Calderón se caractérise par une conscience si exacerbée du problème et par un niveau de réflexion si élevé que ce théâtre présente un intérêt non seulement pour le peuple mais aussi pour les spécialistes de philosophie et de théologie.

Y a-t-il eu un état originel où l'homme vivait isolé et en-deçà de l'état social? Le théologien Suarez avait spéculé au $\mathrm{XVI}^{\mathrm{c}}$ siècle sur l'éventualité d'un status hominis in puris naturalibus voire sur un status pure naturalis. L'espagnol Vives pense que l'homme est certes fait pour la vie en communauté mais que par son égoïsme, il est brutal envers autrui et recherche pour lui-même et pour son propre avantage à s'approprier le plus grand nombre de biens possible, ce qui suscite les plus grands troubles. Dans son Methodus ad facilem historiarum cognitionem paru à Paris en 1566, le sceptique Jean Bodin suppose l'existence d'un état originel caractérisé par une brutalité animale où les hommes vivant dans les bois et dans les campagnes comme des bêtes sauvages ne pouvaient acquérir et posséder que ce qu'ils avaient arraché par la force. C'est justement ce que reprend Hobbes qui séjourne à Paris de 1640 à 1651 lorsqu'il qualifie d'état de nature la situation de l'homme hors de la société, état caractérisé par la guerre de tous contre tous à laquelle seul un contrat social pourrait mettre fin. 
Hobbes est en accord avec Aristote en écrivant que le bonheur de l'homme ne peut être atteint que dans l'État. Toutefois Hobbes corrige Aristote dans la mesure où pour lui l'homme n'est pas par nature un être social, un zoon politicon. Pour Hobbes qui avait publié en 1642 les premières parties de sa principale ouvre politique, les premiers hommes seraient comparables à des champignons qui sortiraient de terre et qui vivraient dans un état de guerre permanent, se combattant les uns les autres dans la pauvreté, la solitude et la violence.

La tension entre état de nature et état social est présente dans le théâtre de Calderón. Nombreux sont les personnages de Calderón comme par exemple Sémiramis dans La hija del aire ou Segismundo dans La vida es sueño qui grandissent loin de la société. Dès qu'ils entrent en contact avec elle, ils apparaissent d'abord aux yeux de la société civilisée comme des sauvages inadaptés. Ceci est particulièrement évident dans la pièce Eco y Narciso présentée par Calderón en 1661. La pièce se joue dans un monde pastoral où la jeune Écho, vénérée par les bergers, s'intéresse à Narcisse que sa mère Liriope a élevé dans les bois, loin de toute communauté humaine. Au fil de la pièce, ce n'est pas Narcisse qui est élevé au niveau d'Écho, mais Écho qui est abaissée au niveau de Narcisse. À cause d'un poison que Liriope donne à Écho de peur qu'elle ne nuise à son fils, celle-ci n'est plus capable que de répéter les dernières syllabes prononcées par une autre personne. Elle perd donc la capacité de communiquer avec les hommes après avoir quitté la communauté des pâtres et s'être rendue sur le territoire sauvage de Narcisse: "Huyendo de los poblados a las asperas montañas iré y, escondida en ellas, las más cóncavas estancias viviré triste y confusa» ${ }^{4}$. Derrière l'aimable monde pastoral, il existe donc un monde sauvage qui est aussi dangereux que nuisible car la vie n'y est ni civilisée, ni sociale, ni ordonnée. Cela devient évident lorsque Liriope se voit qualifiée tantôt d'«ignorada fiera destos montes», tantôt d' "extraño monstruo", d' "especie diferente», ou de "salvaje» ${ }^{5}$, tandis que parmi les bergers règnent la galanterie, la discrétion et le sentiment d'appartenir à une communauté. L'isolement de Narcisse qui vit dans les bois comme un sauvage incapable de communiquer, contraste donc avec la communauté des pâtres, caractérisée par des règles, des mœurs

${ }^{4}$ Calderón, Eco y Narciso. Comedia, vv. 2811-2815.

5 Calderón, Eco y Narciso. Comedia, vv. 366-367; 386; 663; 1305-1306. 
et un discours raffinés, tout comme l'état originel contraste avec l'état social dans la théorie politique.

Si les lois et les décrets édictés par un roi dont le pouvoir est légitimé par la grâce divine doivent être conformes à l'esprit du créateur divin, alors la lex positiva provient de la lex divina. Dans Amar después de la muerte, Calderón montre quels problèmes surgissent lorsque ce n'est pas le cas. Dans la pièce, la révolte et la sécession des Maures sont dues à une ordonnance du roi Philippe II stipulant que désormais plus aucun Maure n'a le droit de célébrer les fêtes nationales, de porter des habits de soie, de se rassembler librement dans la maison de l'un d'entre eux ou de se servir de la langue arabe. Les Maures pensèrent alors que les Chrétiens cherchaient à les réduire en esclavage et ils se retirèrent dans les montagnes de Alpujarra avec des armes et de la nourriture pour y vivre non pas en esclaves mais en hommes libres. Les Maures nommèrent un chef et affrontèrent les Espagnols. Dès le début du drame on insiste sur la coutume mauresque qui prescrit que le vendredi est un jour de fête marqué par des cérémonies religieuses. Par trois fois sont évoqués Allah et le glorieux passé mauresque. Par trois fois les Maures présents s'unissent dans l'appel: «Su ley viva" ${ }^{6}$, et donc suivent la lex divina de l'Islam d'après laquelle il peut être décidé de désobéir à la lex positiva espagnole. Toutefois Calderón laisse le Maure Malek qualifier la loi espagnole de «ley justa y prevención santa» ${ }^{7}$ car il semblait naturel, sans vouloir bousculer les choses, que les mœurs africaines tombaient peu à peu en désuétude. Finalement c'est Isabel Tuzani qui va mettre fin à la révolte, se fiant au pardon général des Espagnols. Si l'on tient compte du fait que, de par son apparence elle est mauresque mais que dans son for intérieur elle est chrétienne, c'est elle qui permet le contrepoint ludique à la situation qui existe au début du drame.

Dans certains cas donc la désobéissance civile à de mauvaises lois est permise. Il est aussi concevable que le système entier du droit positif soit erroné lorsqu'il est par exemple en contradiction avec le bien commun de l'homme ou avec la loi divine. Les lois positives sont également nettement à l'opposé de l'univers du divin lorsqu'elles conduisent à l'athéisme. L'humaniste Luis de León avait signalé en 1571 dans

${ }^{6}$ Calderón, Amar después de la muerte, p. 351.

7 Calderón, Amar después de la muerte, p. 352. 
son De legibus, que la désobéissance civile se justifie quand on est en droit d'affirmer avec certitude que l'obéissance à la loi va à l'encontre du bien commun. Luis de Montesinos qui enseigna à l'université d'Alcalá de 1593 à 1620 donne des raisons supplémentaires à la justification de la désobéissance civile. L'obéissance est certes une prescription provenant d'un droit naturel issu du droit divin, à condition toutefois que le souverain exerce son pouvoir en tenant compte du bien commun, en ne réprimant pas ses sujets et en ne les réduisant pas à l'esclavage. L'obéissance n'est pas une valeur absolue mais dépend de l'exercice du pouvoir. Plus les manquements du pouvoir sont importants, plus la désobéissance civile est justifiée. Dans Quaestio de sedetione, l'humaniste et thomiste Francisco de Vitoria se demande quand l'insurrection du peuple et la révocation du tyran sont justifiables. Il compare une insurrection à une guerre puisqu'il s'agit dans ce cas d'une confrontation avec une attaque et une défense. En se référant à la Bible où l'apôtre Saint Paul s'oppose aux sadducéens et aux pharisiens, il accorde au peuple le droit de mettre fin à une tyrannie. Le droit pour un particulier de tuer un tyran se déduit du droit de la légitime défense contre l'agresseur. PourVitoria, tuer celui qui attaque l'État est alors d'autant plus légitime. Il y a dans La gran Cenobia de Calderón un exemple de tyrannicide justifié. Le tyran Aurelian ne tient pas parole et s'intéresse peu au bien-être de ses sujets. Lorsque Decius l'assassine parce qu'il est barbare, tyrannique, présomptueux et cruel, tous lui en sont reconnaissants et font de lui leur souverain. Aurelian n'avait agit ni selon la lex divina, ni selon la lex naturalis, ni selon la morale, ni pour le bien commun.

La conséquence d'un pouvoir absolu régnant sans prendre en considération les limites de la loi ou de la morale est le cynisme du pouvoir. C'est ce que Thucydide avait illustré à travers une légation d'Athéniens militairement supérieure. Après avoir demandé aux habitants de la petite île de Mélos pourquoi ils ne pourraient pas les asservir, on leur répondit que ceci porterait atteinte à leur réputation, était contraire à leur respectabilité et opposé à la volonté des dieux. Depuis l'Antiquité on conseille donc au souverain de se comporter selon les vertus cardinales de prudentia, iustitia, fortitudo et temperantia, et ceci jusqu'au moment où Machiavel sépare l'exercice du pouvoir et la morale. Lorsque le prince agit sur la base de la morale et doit en même temps conserver son pouvoir, a-t-il le droit de devenir im- 
moral s'il peut ainsi accroître ce pouvoir? Les machiavélistes et les antimachiavélistes ont débattu pour savoir si la raison d'État justifiait l'immoralité. Si l'art de gouverner ne dépend plus de la morale, on peut facilement supposer que le souverain a le droit d'être immoral dans l'intérêt du pouvoir.

La force vertueuse implique une capacité de modération sans laquelle elle mène à la luxure qui d'après Rivadeneira conduit à l'hostilité, au crime, au meurtre et se termine par une atteinte portée à l'État. Calderón en offre un bel exemple dans la pièce intitulée La cisma de Ingalaterra où le roi Henri VIII, à la vue d'Anne Boleyn, renonce à ses études de théologie, perd l'entendement aveuglé par l'amour, prend des décisions fatales telles que divorcer de sa première femme et se marier avec Anne, expulser Wolsey, et ordonner que l'Église anglicane se sépare de l'Église catholique. Il impose cette dernière mesure avec une extrême brutalité, menaçant de peine de mort quiconque contesterait sa décision. Anne, qui en aime un autre, simule son amour pour le roi, sur les conseils du cardinal Wolsey qui, assoiffé de vengeance, espère par là obtenir la protection d'Anne. Cependant Anne ne récompensera pas Wolsey. Ingrate et tyrannique, elle le repoussera cruellement. À la fin de la pièce, quand il ne reste plus comme future souveraine que Marie, la fille d'Henri, et que celle-ci doit reconnaître devant le parlement la dépossession de l'Église catholique par les anglicans, elle opposera la loi divine à la raison d'État: «Y pues vuestra majestad sabe la verdad, no quiero que por razones de Estado la ley de Dios se pervierta» ${ }^{8}$. Tandis que le roi sollicite que l'on soit plein d'indulgence devant l'inexpérience de la jeune princesse, il est cependant clair pour le public que la lex divina doit se situer au-dessus de la raison d'État.

Puisque dans les monarchies du début des temps modernes la politique était le fait des princes et des rois, elle était donc toujours intimement liée à l'éthique. Malgré tout dans certains cas, Calderón prend pour sujet un comportement juste, indépendamment de la politique. Dans ce contexte nous pouvons recourir à Eco y Narciso, puisque la pièce interroge les conditions du bonheur et du malheur. Partant du principe que la raison a le bonheur pour but, l'absence de bonheur résulte donc d'un manque de raison. Pour Saint Thomas

${ }^{8}$ Calderón, La cisma de Inglaterra, p. 520. 
d'Aquin le malheur était le résultat d'actes humains involontaires provenant de la violence, de la peur et de l'ignorance, mais pas de la raison. Pour lui en effet, il y a des sentiments positifs ou négatifs comme l'espoir, la peur, la douleur, la haine et l'amour qui sont classés soit dans le domaine du plaisir soit dans celui de la souffrance. Dans Eco $\gamma$ Narciso, le plaisir domine dans le monde bucolique d'Écho pendant que le déplaisir l'emporte chez Narcisse. En raison d'une prophétie le concernant, Liriope a peur pour son fils. Il fait de cette peur la sienne, et vit redoutant une voix une beauté. Il fuit Écho et tombe amoureux de sa propre image. C'est donc d'abord la peur de Liriope, puis l'ignorance de Narcisse qui conduisent à des actes déraisonnables et aboutissent finalement au malheur. Par la suite, la peur s'empare aussi d'Écho lorsque Narcisse ne répond pas à son amour. L'impossibilité de parler et par conséquent de s'exprimer raisonnablement en société est le résultat du poison que lui a donné Liriope. Sa vie ultérieure restera marquée par la tristesse et par le malheur. Calderón montre ainsi que la connaissance de soi et la connaissance de l'autre ne sont pas indépendants des sentiments. Narcisse est présenté dès le début comme un personnage ignorant. Écho perd la faculté de s'exprimer au contact de Liriope et de Narcisse. Narcisse offre un bel exemple de l'effet produit par des sentiments négatifs qui rendent impossibles la connaissance de soi et la formation de l'identité. La même chose se passe pour Écho qui, infectée par Narcisse, «se ha convertido en aire» ${ }^{9}$. Calderón montre ainsi comment l'ignorance donne naissance à des actes involontaires et combien ceux-ci accompagnés de sentiments négatifs conduisent au malheur. En exposant les conséquences d'un comportement incorrect, il éduque le spectateur et le conduit à adopter un comportement juste.

Narcisse ne pouvait donc tirer aucun savoir de son univers, car il avait grandi loin de la société, dans les forêts et les montagnes. Segismundo de La vida es sueño apparaît tout autant éloigné de la société, car il a grandi dans une tour isolée. Certes il avait son précepteur Clotaldo, mais il n'a pas pu expérimenter pratiquement le comportement convenable, ni donc de s'y habituer et d'en faire sa seconde nature. On remarque à quel point le monde lui est étranger lors de sa première rencontre avec Rosaura, pendant laquelle il dit en savoir

9 Calderón, Eco y Narciso. Comedia, p. 43. 
très peu sur le monde, puisque la tour est à la fois son berceau et son tombeau. Lorsqu'il qualifie l'endroit de sa captivité de "désert campagnard", il fait allusion à la tabula rasa de son monde empirique. Il est clair que l'habitude des rapports sociaux lui fait défaut quand il se dépeint comme un "hombre de las fieras y una fiera de los hombres»" Ce qu'il sait de la politique, il l'a appris des animaux. Ce sont les oiseaux et les bêtes sauvages qui lui ont enseigné la rhétorique. Il n'est donc pas étonnant que lors de sa première visite au palais il soit plein d'admiration et d'étonnement, qu'il ne se contrôle plus et veuille jeter par la fenêtre quiconque se trouve sur son chemin. On insiste sans cesse sur le fait que Segismundo a grandi parmi les animaux. Luimême se voit comme un amalgame fait d'animal et d'être humain. Rosaura refuse même de reconnaître en lui toute nature humaine. Ayant grandi parmi les bêtes il lui apparaît comme un être grossier, cruel, arrogant, barbare et tyrannique. Par conséquent ses actes sont qualifićs de cnojo, rigor, rabia et cólcra.

Ajoutons que depuis l'Antiquité nous nous représentons couramment l'homme, comme ayant, contrairement à l'animal, une seconde nature en plus de sa première nature physique. À la physis (nature) s'ajoute donc l'hexis (habitude). Selon Aristote, les vertus morales, contrairement aux capacités théoriques, ne pourraient se développer que grâce à un entraînement pratique préalable. Seul celui qui s'est exercé à la vertu de l'équité est juste. Seul celui qui s'est habitué à mépriser le danger est brave. La vertu dépend donc du développement d'un habitus qui enseigne comment conduire les émotions et par exemple, dans le cas de la colère comment éviter une réaction extrême au profit d'une réaction mesurée. La vertu est donc un habitus qui oriente l'action vers le bien. La scolastique ainsi que la néo-scolastique ont adopté cette position aristotélicienne. Le fondateur de l'ordre des Jésuites Ignace de Loyola confirme lui aussi cette position quand il parle de "buenos hábitos» et de "sólidas virtudes»" ${ }^{11}$. L'échec de Segismundo entrant pour la première fois dans le palais, n'est donc pas dû à une prophétie funeste, mais à l'absence d'un habitus vertueux qu'il n'aurait pu acquérir que par la pratique. Sans cet habitus, une seconde nature lui fait défaut de sorte qu'il ne peut agir qu'avec la

${ }^{10}$ Calderón, La vida es sueño, p. 41.

11 Ignacio de Loyola, Obras completas, p. 612. 
sauvagerie des animaux qui l'ont entouré. C'est sa première entrée au palais qui sera d'un seul coup l'expérience qui va lui permettre de changer. Ainsi Calderón exige-t-il que les vertus deviennent, par la pratique, des habitudes.

Calderón présente des arguments tout aussi subtils dans les questions théologiques, comme par exemple celle de la Trinité. Le dogme de la Trinité permet à Dieu de se faire homme en Jésus sans pour cela perdre de sa divinité. Le christianisme n'est donc ni tout à fait monothéiste, ni tout à fait polythéiste mais postule l'existence d'un dieu dans lequel se rejoignent l'unicité et la pluralité. Comme ce postulat n'est pas compatible avec la logique aristotélicienne, il y a eu dans les premiers siècles après Jésus-Christ de nombreuses tentatives d'interprétation qui pour la plupart ont été plus tard rejetées comme hérésies par l'Église.

Dans son auto sacramental $A$ diós por razón de Estado comme aussi dans d'autres pièces, Calderón présente différents aspects de la sainte Trinité. Il tient compte de l'unité de l'essence (unitas in trinitate), de l'unicité dans l'action exercée vers l'extérieur (unitas operationis ad extra) et des rapports au sein de la divinité (unitas circumincessionis). Le Père, le Fils et le Saint Esprit ne sont pas trois natures différentes mais une seule et même essence. Calderón refuse le polythéisme de l'Antiquité en faisant remarquer que les dieux antiques étaient des individus agissant pour la plupart les uns contre les autres et même immoralement. Dans de nombreux passages, il oppose au polythéisme le Dieu chrétien comme étant un «Diós, en esencia uno, y en persona tres»" ${ }^{12}$ ou "según la $\mathrm{Fe}$ informa, es en la Esencia Uno y Trino en las personas» ${ }^{13}$. Dans Las cadenas del Demonio San Bartolomé donne une explication dogmatique précise:

Este es único en esencia y trino en personas, pues el Padre, que es la primera, ni criado ni engendrado, ni procedido se ostenta de nadie, porque en Sí mismo sin fin ni principio reina. El Hijo, que es la segunda desta soberana esencia, ni criado ni procedido, sino engendrado se muestra del Padre, cujo concepto siempre incesable se engendra. El Espíritu, que es de aquesta esencia suprema la tercera, ni criado ni engendrado, cs

12 Calderón, Amar y ser amado y divina Filotea, p. 1786.

13 Calderón, El divino Orfeo, p. 1844. 
cosa cierta, sino procedido de ambos; que aunque tres personas sean, nos son tres dioses; uno solo Dios es no más, una mesma voluntad, un querer mismo y una misma omnipotencia ${ }^{14}$.

De plus, dans la Bible on attribue la toute puissance au Père, l'omniscience au Fils, la bonté et le salut au Saint-Esprit, ce que Calderón formule de la façon suivante: "de manera que en la comunicatión de personas, dando en ellas al Espíritu el Amor, al Padre la Omnipotencia y la Sabidiría al Hijo" "15. Mais afin que l'unicité soit respectée on peut lire dans $A$ Dios por razón de Estado: "Pues una en los tres la deidad, uno en los tres el poder, uno en los tres el amor y uno en los tres el saber, cierto que en la esencia es uno, siendo en las personas tres» ${ }^{16}$. Toutefois dans la même pièce l'unicité du Fils et du Père est ressentie comme un problème: "¿Que si adora (como dijo) sólo un Dios, cómo después dijo que a su Hijo esperaba? ¿Hijo y Padre fuerza no es que sean dos? ¿Pues cómo a uno adora sólo?»17 I'arianisme considérait le Fils comme création du Père ce qui, par conséquent lui interdisait l'éternité. Le dogme de l'Immaculée Conception est d'un grand secours pour comprendre la double nature du Christ, humaine et divine, ce sur quoi Calderón insiste: "encarnado en una Virgen madre, que antes y después y entonces permaneciese siempre Virgen» ${ }^{18}$. À l'issue de ses argumentations compliquées et exigeantes pour l'entendement humain en faveur de la Trinité, Calderón écrit une phrase qu'il accompagne musicalement: "Gran Dios que ignoramos, abrevia el tiempo y haz que te conozcamos, pues te creemos»" ${ }^{19}$. Quelle leçon donne ainsi Calderón à ses spectateurs? À la fin de la pièce il déconseille de s'abandonner à une résignation sceptique, face à des arguments compliqués exposés dans leurs moindres détails, au profit d'une vie pragmatique s'appuyant sur les dix commandements et surtout la foi et l'amour: "que debe el ingenio humano llegarlo a amar y creer por razón de Estado cuando faltara la fe» ${ }^{20}$. Le fait qu'ici en-

14 Calderón, Las cadenas del Demonio, p. 1255.

15 Calderón, La protestación de la Fe, p. 732.

16 Calderón, $A$ Dios por razón de Estado, p. 865.

17 Calderón, $A$ Dios por razón de Estado, p. 864.

18 Calderón, $A$ Dios por razón de Esiado, p. 865.

19 Calderón, $A$ Dios por razón de Estado, p. 850.

${ }^{20}$ Calderón, $A$ Dios por razón de Estado, p. 869. 
core la raison d'État soit évoquée boucle nos argumentations. Ce mot clé de Machiavel, dans l'interprétation qu'en ont fait les Jésuites Rivadeneira et Mariana qui unissent la morale et le maintien du pouvoir, apparaît aussi judicieux quand il s'agit de la razón de estado de tí mismo, qui selon Gracián protège et sauvegarde l'individu. Ici comme dans l'auto sacramental El gran teatro del mundo, Calderón conseille un obrar bien et clôt ses argumentations théologiques d'une façon pédagogiquement sensée par des instructions pratiques.

En résumé nous pouvons voir que le théâtre du siècle d'or espagnol répond à des problèmes politiques fondamentaux en thématisant l'état originel présocial, la tension entre la lex positiva et la lex divina, la raison d'État, la révolte du peuple et le tyrannicide. Nous pouvons retenir que Calderón prend en considération les problèmes éthiques en rapport avec le bonheur, l'ignorance, ou les passions et les habitus néfastes. Il se penche sur de difficiles questions théologiques comme la question de la sainte Trinité. Ce faisant il offre à ces problèmes des solutions d'une grande valeur pédagogique. 


\section{Bibliografía}

ARistóteles, Retórica, Madrid, Aguilar, 1968.

- Nikomachiscke Ethik, Hamburg, Mciner, 1995.

- Metafisica, Madrid, Grcdos, 1998.

- Acerca del alma, Madrid, Gredos, 1999.

- Ética nicomáquea, Madrid, Gredos, 2000.

- Tratados de lógica (Órgano) / 1: Categorías, Tópicos, Sobre las refutaciones sofisticas, Madrid, Gredos, 2000.

- Politik, ed. O. Gigon, Düsseldorf, Artemis und Winkler, 2006.

Calderón do la Barci, P., $A$ Dios por razón de Estado, en Obras Completas, Autos sacramentales, III, ed. Á. Valbuena Prat, Madrid, Aguilar, 1952.

- Amar después de la muerte, en Obras completas, ed. L. Astrana Marín, Madrid, Aguilar, 1951, vol. I.

- Amar y ser amado y divina Filotea, en Obras completas, Autos sacramentales, III, ed. Á. Valbuena Prat, Madrid, Aguilar, 1952.

- Eco y Narciso. Comedia, ed. Ch.V. Aubrun, Paris, Centre de Recherches de l'Institut d'Études Hispaniques, 1963.

- El divino Orfeo, en Obras Completas, Autos sacramentales, III, ed. Á. Valbuena Prat, Madrid, Aguilar, 1952.

- La cisma de Inglaterra, en Obras completas, ed. L. Astrana Marín,Madrid, Aguilar, 1951, vol. I.

- La gran Cenobia, en Obras completas, ed. L. Astrana Marín, Madrid, Aguilar, 1951, vol. I.

- La protestación de la Fe, en Obras Completas, Autos sacramentales, III, ed. Á. Valbuena Prat, Madrid, Aguilar, 1952.

- La vida es sueño, cd. D. Ynduráin, Madrid, Alianza, 2002.

- Las cadenas del Demonio, en Obras Completas, Dramas, I, ed. Á. Valbuena Briones, Madrid, Aguilar, 1951.

Gracián, B., Obras completas, Madrid, Aguilar, 1960.

- Oráculo manual $y$ arte e prudencia, Madrid, Turner, 1995.

Hobizes, Th., Vom Menschen. Vom Bürger, ed. G. Gawlick, Hamburg, Meiner, 1966.

- Vom Körper, Hamburg, Felix Meiner, 1967.

- Leviathan, ed. H. Klenner, Hamburg, Mciner, 1996.

LEón, L. de, De legibus, Madrid, CSIC, 1963.

LOYOLA, I. de, Obras completas, Madrid, BAC, 1952.

Maguiavelo, Der Fürst [El príncipe], Stuttgart, Reclam, 1986.

Maravall, J. A., Teatro y literatura en la sociedad barroca, Madrid, Seminarios y ediciones, 1972.

MarianA, J. de, "Del rey y de la institución real», en Obras, ed. F. Pi y Margall, Madrid, Atlas, 1950, vol. II, pp. 463-576. 
Rivadineirn, P. dc, "Tratado de la religión y virtudes que debe tener el príncipe cristiano", en Obras escogidas, cd. V. de la Fuente, Madrid, Hernando, 1919, pp. 449-587.

SuÁrfz, F., De legibus I, Madrid, CSIC, 1971.

Thomae Aguinalis, S., Summae contra gentiles, libri quatuor, ed. J.-P. Migne, Paris, 1863.

- De veritate catholicae fidei contra gentiles, libri quatuor, Luxemburg, Brüick, 1881.

- In decem libros ethicorum Aristotelis ad Nicomachum expositio, Roma, Marietti, 1949.

- Summa Theologica, ed. Philosophische-Theologische Hochschule Walberg bei Köln, Heidelberg / Graz / Wien / Köln, Gemeinschaftsverlag F.H. / Verlag Styria, 1953-1977.

- Über die Herrschaft der Fürsten, Stuttgart, Reclam 1971.

Thucydides, Geschichte des Peloponnesischen Krieges, München, DTV, 1977.

Vitoria, F. de, Relectio de Iure Belli o Paz Dinámica, Madrid, CSIC, 1981.

Vives, J. L., Tratado del alma, Buenos Aires, Espasa Calpe, 1945.

- Über die Gründe des Verfalls der Künste. De causis corruptarum artium, ed. E. Hidalgo-Serna, München, Fink, 1990. 